\section{BS13 ROLE FOR MICRORNA-26B IN VESSEL CALCIFICATION}

${ }^{1}$ David Mellis*, 'Andrea Martello, 'Mark MacAskill, 'Loes Elemans, 'Souzana Angel, ${ }^{2}$ Ayman Al-Haj Zen, 'Adriana Tavares, 'Rachael Forsythe, 'Ross Jones, ${ }^{1}$ Brad Wagner, ${ }^{4}$ David Newby, ${ }^{1}$ Andrew Baker, ${ }^{1}$ Tijana Mitic, ${ }^{1}$ Carlos Corral, ${ }^{3}$ Michael Robinson, ${ }^{1}$ Andrea Caporali. ${ }^{1}$ University of Edinburgh; ${ }^{2}$ University of Oxford; ${ }^{3}$ Miami University; ${ }^{4}$ British Heart Foundation Centre for Cardiovascular Science, University of Edinburgh

10.1136/heartjnl-2019-BCS.176

Introduction MicroRNAs (miRNAs) are small non-coding RNAs that orchestrate genetic networks by modulating simultaneous gene expression and regulates vascular function. Despite recent accumulation of experimental data and the emergence of functional models, the complexity of miRNA-based regulation is still far from being well understood. By performing high-content phenotypic screening (HCS), miR-26b emerged from the screen as a top candidate for ECs proliferation. The overexpression of miR-26b significantly increased EC proliferation, migration and survival. Furthermore, miR-26b mimics increased EC tube formation and branching morphogenesis. HCS and bioinformatic analysis identified both Phosphatase and Tensin homolog (PTEN), a gene critical in cell survival and Bone Morphogenetic Protein Receptor Type 1B (BMPR1B; ALK6), important in calcium homoeostasis, as direct targets of miR-26b. Because BMPR1B was identified as a direct target of mir-26b, we decided to study the role of miR-26b in vessel calcification. To test our hypothesis, miR-26b CRISPR/Cas9 global knock-out (KO) mice were generated.

Objective The aim of this study is to characterize how miR26b can influence vascular calcification and characterize ALK6 as mir-26b target.

Methods In this study, we have used HCS and miR-26b KO mice and artery from patients with critical limb ischaemia to prove our hypothesis

Results We confirmed that miR-26b was knocked down in the miR-26b KO mice without effecting the expression of other miR-26 family members and the host gene. To test whether miR-26b KO mice were more susceptible to calcification, we extracted the heart and aorta from 3-month-old mice and cultured for 6 days in a high phosphate media with and without warfarin. Histological analysis with the calcium stains von Kossa and alizarin red confirmed that miR-26b KO mice displayed a higher level and susceptibility to calcifying compared to control mice. Expression of the markers of calcification (RUNX2 \& ALK6) supported the histology. Immunohistochemistry and qPCR confirmed that the expression of ALK6 was also increased in the miR-26b KO. To further characterise this phenotype, mice were aged and multiple tissues including the aorta were harvested from 3 and 6 month old mice. The aorta and hearts were then prepared for high resolution PET/ CT using the highly calcium specific sodium fluoride (18F) probe. This probe allows for the detection of both micro and macro calcification. PET/CT supported our ex-vivo culture model and confirmed that 6-month-old miR-26b KO mice had a higher level of micro-calcification compared to control mice. To investigate the clinical relevance of miR-26b, we analysed artery and muscles from below the knee amputated limbs from patients with critical limb ischaemia. The levels of miR$26 \mathrm{~b}$ was decreased in the muscle biopsy from the less perfused section of the amputated leg compared to more perfused counterpart. Moreover, miR-26b was downregulated in the calcified tissue portion of the artery in comparison to the soft tissue.
Conclusion Taken together these findings demonstrate an important role for miR-26b in vessel calcification and suggest its effects are through the upregulation of BMPR1B; Alk6. Conflict of interest None

\section{BS14 C-REL DRIVES ATHEROSCLEROSIS AT SITES OF DISTURBED BLOOD FLOW BY ACTIVATING INFLAMMATORY AND PROLIFERATIVE TRANSCRIPTIONAL PROGRAMMES IN ENDOTHELIUM}

${ }^{1}$ Blanca Tardajos Ayllón*, ${ }^{1}$ Celine Souilhol, ${ }^{2}$ Fiona Oakley, ${ }^{1}$ Paul Evans. ${ }^{1}$ University of Sheffield; ${ }^{2}$ Newcastle University

\subsection{6/heartjnl-2019-BCS.177}

Introduction Atherosclerosis is an inflammatory disease that develops preferentially at bends and branches of the vasculature exposed to disturbed flow and low shear stress (LSS). Shear stress modifies endothelial cell (EC) function by regulating proliferation, inflammation and other fundamental processes. Shear stress alters multiple transcriptional programs, including those regulated by the NF- $\mathrm{B}$ family of transcription factors. Although some members of the NF- $\kappa \mathrm{B}$ pathway are known to respond to shear, the influence of haemodynamics on the $\mathrm{c}-\mathrm{Rel} \mathrm{NF}-\mathrm{\kappa B}$ subunit and its role in atherogenesis are unknown, and are a focus of the current study.

Methods C57BL/6 wildtype and c-Rel knockout mice were used to quantify the expression of c-Rel, the inflammatory protein E-selectin, and EC proliferation at LSS and high shear stress (HSS) regions of the aorta. To establish the role of cRel in atherosclerosis, C57BL/6 wildtype and c-Rel knockout mice were treated with AAV-PCSK9 and fed a high fat diet for 6 weeks. Plaque burden was quantified using Oil Red O. In vitro studies were performed using human umbilical vein EC (HUVEC) or human coronary artery EC (HCAEC) exposed to LSS or HSS. c-Rel was silenced using siRNA and EC proliferation and inflammation were measured by PCNA staining and qRT-PCR, respectively. Gene expression was studied using a microarray (ClariomTM S).

Results En face staining of murine aortas revealed a striking enrichment of $c-R e l$ at LSS regions $(\mathrm{P}<0.01)$. Similarly, c-Rel expression was significantly enhanced in HUVEC or HCAEC exposed to LSS compared to HSS $(\mathrm{P}<0.05)$. c-Rel genetic deletion in mice resulted in decreased proliferation and inflammation in EC at LSS sites, and it also decreased lesion area in AAV-PCSK9-treated mice $(\mathrm{P}<0.05)$. Moreover, depletion of c-Rel by siRNA in HUVEC resulted in decreased proliferation and inflammation under LSS $(\mathrm{P}<0.05)$. Microarray studies using HUVEC revealed that c-Rel activates genes implicated in inflammation and proliferation, including components of MAPK and non-canonical NF- $\kappa$ B pathways.

Conclusion s.

Our data demonstrate that c-Rel promotes EC pathophysiological changes at LSS regions and is a driver of atherosclerosis. Studies in cultured EC revealed that c-Rel activates numerous genes including components of MAPK and non-canonical NF- $\mathrm{KB}$ pathways, thereby providing a potential mechanism for its proinflammatory and proproliferative effects.

Conflict of interest None 